\title{
SÍNTESE VERDE DE 1,3-DIARILTRIAZENOS SIMÉTRICOS E ASSIMÉTRICOS EM VINAGRE
}

\author{
Leonardo C. Messina ${ }^{a}$ e Álvaro T. Omoria,*,(1) \\ ${ }^{a}$ Centro de Ciências Naturais e Humanas, Universidade Federal do ABC, 09210-580 Santo André - SP, Brasil
}

Recebido em 20/08/2020; aceito em 15/09/2020; publicado na web em 15/10/2020

\begin{abstract}
SYNTHESIS OF SYMMETRIC AND ASYMMETRIC 1,3-DIARYLTRIAZENES IN VINEGAR. An adaptation on the classical synthesis methodology for 1,3-diaryltriazenes for a 4-hour experimental class has been proposed with green chemistry principles in mind by using commercial vinegar as a source for diazotization with $\mathrm{NaNO}_{2}$ and keeping the reaction workup to simple vacuum filtration. Several anilines with different substituent groups were used to evaluate how they affect the synthesis based on their electron-donating/withdrawing characteristics. The decomposition of the triazenes in acidic media was also monitored via TLC. On the overall, the reactions came up with medium to high yield and high purity. Experimental procedure, reaction mechanism and other potential discussions such as green chemistry principles and effects of electron-donating/withdrawing groups are suitable for undergraduate students.
\end{abstract}

Keywords: organic chemistry; green chemistry; anilines; triazenes; vinegar.

\section{INTRODUÇÃO}

Nucleofilicidade, basicidade e cinética são conceitos fundamentais da química e possuem uma forte correlação entre si. O entendimento de tais conceitos se faz necessários para entender como, por que e com qual velocidade uma determinada reação química ocorre e isto para diversos tipos de reação: substituição eletrofílica ou nucleofílica, reações de adição ou eliminação, reações de condensação, reações ácido-base, enfim, uma ampla variedade. Dessa forma, o ensinamento de tais conceitos aos alunos de graduação se faz um pilar de sustentação para o aprendizado de conceitos mais avançados no futuro.

Sabe-se que, costumeiramente, alguns conceitos da química podem aparecer um tanto abstratos e não tão bem consolidados apenas com aulas teóricas. No entanto, a química dispõe de diversas ferramentas para contornar isso, uma delas é o fato de ser uma disciplina experimental, o que agrega no ensinamento aos alunos, uma vez que eles podem por si mesmos verificar, testar e comprovar os conhecimentos teóricos, na prática.

Uma classe de moléculas capaz de ensinar tais conceitos de química são os triazenos. Triazenos são compostos de cadeias lineares caracterizados por três nitrogênios ligados em sequência, possuindo uma fórmula geral $\mathrm{RN}=\mathrm{N}-\mathrm{NR}$ 'R'. Já os 1,3-diariltriazenos são triazenos que possuem agrupamentos arila $\left(R, R^{\prime}=A r\right)$ em suas extremidades. Os triazenos possuem atividade biológica atuando como pró-fármacos, ${ }^{1}$ sendo a dacarbazina ${ }^{\circledR}$ (Figura 1-A) e temozolomida (Figura 1-B) utilizadas como antitumorais, além de apresentarem atividade antibacteriana e antifúngica, ${ }^{2,3} \mathrm{O}$ diminazeno<smiles>CN(C)/N=N/c1[nH]cnc1C(N)=O</smiles>

A<smiles>Cn1nnc2c(C(N)=O)ncn2c1=O</smiles>

B
(Figura 1-C) é utilizado no tratamento da babesia, doença protozoária que afeta animais de pecuária e domésticos. ${ }^{4,5}$

Outra forma de utilização desses compostos é na determinação espectrofotométrica de metais, uma vez que possuem propriedades complexantes. ${ }^{6} \mathrm{O}$ hidrogênio da estrutura triazênica é relativamente ácido $\left(\mathrm{pK}_{\mathrm{a}} \text { entre } 9,5 \text { e } 14\right)^{4,6}$ e na desprotonação gera uma estrutura com carga deslocalizada e um excelente ligante monodentado ou agente quelante e que também pode atuar com ponte entre centros metálicos (Figura 2). ${ }^{4}$

Triazenos também são utilizados na síntese orgânica, podendo atuar na síntese de 1,2,3-triazóis, éteres, biarílicos, dentre outros. ${ }^{7}$ Triazenos podem ainda servir como bloco base para outras reações, como reações de alquilação no nitrogênio ( $\mathrm{ArN}=\mathrm{N}-\mathrm{NRAr}, \mathrm{R}=$ alquil $){ }^{8}$

A síntese dos triazenos geralmente é via diazotação de anilinas, que ocorre em meio ácido com uso de nitrito orgânico ou inorgânico. Protocolos de diazotação costumeiramente utilizam ácidos minerais fortes, como $\mathrm{HCl}$ e $\mathrm{H}_{2} \mathrm{SO}_{4}$ como fonte de prótons para a diazotação, enquanto métodos mais brandos podem envolver o uso de ácido acético glacial. ${ }^{2}$ Embora sejam métodos eficientes, a presença de ácidos concentrados, posteriormente diluídos ou não, apresenta um risco desnecessário em um protocolo de diazotação, uma vez que recentemente foi observado que o uso de vinagre comercial, uma solução aquosa diluída de ácido acético, é o suficiente para realizar a diazotação, ${ }^{9,10}$ representando um método mais verde para a diazotação, uma vez que apresenta baixa periculosidade, não gera resíduo tóxico por si só, advém de fonte renovável, possui baixo custo e disponível em qualquer mercado.

Além do nitrito de sódio, há reagentes de diazotação orgânicos,
Figura 1. Estrutura da dacarbazina ${ }^{\circledR}(A)$, da temozolomida $(B)$ e do diminazeno $(C)$<smiles>N=C(N)c1ccc(/N=N/Nc2ccc(C(=N)N)cc2)cc1</smiles> 
Monodentado<smiles>[R]N=NN([R2])C</smiles>

Quelato<smiles></smiles>

Em ponte<smiles>[R]/[Y1]=N\N([R])[M]</smiles>

Figura 2. Modos de coordenação mais comuns para os triazenos ${ }^{4}$

como o nitrito de isoamila, entretanto, esse método envolve o uso de solventes orgânicos, como metanol, hexano ou benzeno que, de maneira geral, aumenta os custos de produção e geram resíduos mais tóxicos. ${ }^{11,12}$ Diazotação utilizando sistema de $\mathrm{CO}_{2} /$ $\mathrm{H}_{2} \mathrm{O}$ já foi investigado ${ }^{13} \mathrm{e}$, embora seja um excelente método verde, a metodologia demanda um maior tempo de execução, uso de equipamento especializado, além de um sistema pressurizado de $\mathrm{CO}_{2}$, o que deve ser evitado quando se pretende executar um experimento em um laboratório de graduandos.

O mecanismo de diazotação é bem conhecido e utilizado em diversos tipos de reações, como a de Sandmeyer, Balz-Schiemann e Gomberg-Bachmann. ${ }^{14}$ No caso dos 1,3-diariltriazenos, após a formação do sal de arildiazônio $\left(\mathrm{ArN}_{2}{ }^{+} \mathrm{X}^{-}\right)$, há o ataque nucleofílico da anilina ao diazônio, seguida da abstração de próton, levando à formação do diariltriazeno ArN=N-NHAr' (Figura 3).

Como existe uma enorme variedade de anilinas substituídas (nitro-, ciano-, haloanilinas, anisidinas, etc), torna-se possível realizar sínteses comparativas. Esse experimento comparativo pode indicar como determinado grupo substituinte afeta a síntese em diferentes partes do mecanismo. Por exemplo, a presença de um grupo nitro $\left(-\mathrm{NO}_{2}\right)$ na posição para- pode afetar a diazotação pois a nitroanilina é menos básica. No entanto, uma vez formado o sal de diazônio, derivado da nitroanilina, este-se tornaria mais susceptível a um ataque para a formação do triazeno. Assim, a diferença no rendimento na síntese destes compostos pode ser explicado usando fatores tanto de basicidade como de nucleofilicidade.

A nucleofilicidade geralmente é confundida com a basicidade, porém há suas diferenças. A nucleofilicidade é um conceito cinético que leva em conta a reatividade, já a basicidade é um conceito termodinâmico, relacionado com o equilíbrio químico entre espécies em uma reação com um próton. A basicidade é relacionada com a estabilidade da a espécie, ou então o quão propício a espécie é de abstrair um próton a fim de obter uma melhor estabilidade. Já a nucleofilicidade de um nucleófilo (uma base de Lewis), depende não só de sua basicidade, mas também de outros fatores como efeitos de solvatação e estéricos. ${ }^{15}$

\section{Objetivos pedagógicos}

Este trabalho tem como foco a proposta de um experimento de síntese verde de 1,3-diariltriazenos, um grupo de moléculas que pode introduzir ou reforçar aos alunos alguns princípios de química verde como:

- Uso de solventes seguros;

- Uso de matéria-prima de fonte renovável;

- Prevenção de resíduos, ao apresentar uma metodologia de alta pureza que dispensa etapas de purificação como cromatografia em coluna.
Enquanto no âmbito da química orgânica, os objetivos incluem:

- Praticar a montagem de aparatos básicos de laboratório;

- Como utilizar a técnica de CCD e o equipamento de ponto de fusão;

- Praticar análise de espectros de RMN de compostos simétricos e não-simétricos;

- Descrever mecanismos de reações utilizando setas curvas;

- Entender como grupos doadores/retiradores de elétrons podem afetar a síntese (reatividade) e o produto final (estabilidade);

- Praticar a escrita de relatórios e de como buscar informações relevantes na literatura.

\section{PARTE EXPERIMENTAL}

Todo o procedimento pode ser executado pelos alunos sem a necessidade de serem supridos com alguma solução previamente preparada caso um banho ultrassônico seja disponibilizado. Anilinas solubilizam lentamente devido ao fraco meio ácido proporcionado pelo vinagre, o que pode levar algum tempo sem um banho ultrassônico. Um período de 4 horas de laboratório são o suficiente para executar o experimento. Devido à facilidade do procedimento experimental, é recomendado que os alunos sejam separados em no máximo trios, a fim de maximizar a quantidade de reações diferentes, o que facilita a discussão de resultados. Não se espera que todos os compostos sejam sintetizados em um experimento de graduação, mas a escolha de compostos chaves pode ampliar as discussões. É preferível que os resultados de cada grupo sejam compartilhados com a turma.

\section{Materiais e equipamentos}

Reagentes (Sigma Aldrich ${ }^{\circledR}$ ) e solventes (grau P.A. - Synth ${ }^{\circledR}$ ) foram utilizados sem purificação prévia. Em um mercado local, foi obtido o vinagre (marca: Carrefour, tipo: vinagre de álcool, teor de ácido acético: 4\%). As análises de cromatografia em camada delgada (CCD) foram efetuadas em cromatofolhas de alumínio (gel de sílica 60), com espessura de $0,2 \mathrm{~mm}$, contendo indicador para $254 \mathrm{~nm}$ usando mistura hexano/acetato de etila como fase móvel. As análises de espectrometria de massas de alta resolução (EMAR) foram feitas em um MicroToF Bruker Daltonics (ESI-TOF). As medidas de ponto de fusão foram feitas no equipamento EZ-Melt da Stanford Research Systems ${ }^{\circledR}$, com resolução de $0,1{ }^{\circ} \mathrm{C}$. Espectros de $\mathrm{RMN}{ }^{1} \mathrm{H}$ dos compostos foram obtidos com o equipamento Bruker AIII $500 \mathrm{MHz}$ da Central Analítica da USP e no Varian $(500 \mathrm{MHz})$ da Central Experimental Multiusuário da UFABC.

\section{Procedimento geral da síntese de 1,3-diariltriazenos simétricos}

Em um balão de fundo redondo de $100 \mathrm{~mL}$, foram adicionados $30 \mathrm{~mL}$ de vinagre de álcool e $1 \mathrm{mmol}$ da anilina. A dissolução em vinagre foi forçada com a ajuda do banho ultrassônico. Sobre o agitador magnético, o balão foi deixado sob agitação vigorosa em banho de gelo. Após cinco minutos em gelo, o nitrito de sódio (0,5 mmol) foi adicionado à solução e o sistema reacional foi agitado durante quinze minutos (60 minutos para nitroanilinas). $\mathrm{O}$ banho de

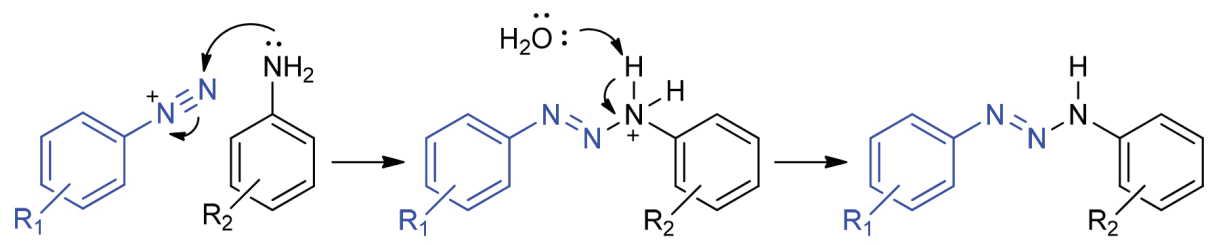

Figura 3. Mecanismo de formação dos 1,3-diariltriazeno 
gelo foi então removido. O procedimento é então dividido em dois métodos, a depender da anilina utilizada na reação.

\section{Método A}

A reação foi mantida a temperatura ambiente por duas horas ou até a formação de um precipitado.

\section{Método B}

Logo após a remoção do banho de gelo, foi adicionado uma solução aquosa de acetato de sódio (15 g em $20 \mathrm{~mL}$ ) e mantido sob agitação em temperatura ambiente por duas horas ou até a formação de um precipitado.

Independente do método utilizado, a mistura reacional é passada para um filtro com placa sinterizada ou funil de Buchner e filtrado sob vácuo. O sólido é seco sob pressão reduzida. Após análise por CCD (cromatografia em camada delgada) o rendimento foi determinado e foi submetido à caracterização por RMN e EMAR.

Cristalização do produto pode levar a melhores resultados de ponto de fusão, mas não foi realizada neste trabalho e não interfere com os objetivos pedagógicos do experimento.

\section{Procedimento geral da síntese de 1,3-diariltriazenos não-simétricos}

A anilina (1 mmol) foi adicionada em um balão de fundo redondo contendo $30 \mathrm{~mL}$ de vinagre e a dissolução foi forçada com a ajuda do banho ultrassônico. Sobre o agitador magnético, o balão foi agitado em banho de gelo e deixado sob agitação vigorosa. Após cinco minutos em gelo, $1 \mathrm{mmol}$ de nitrito de sódio $(69 \mathrm{mg})$ foi adicionado à solução. Após quinze minutos em banho de gelo (60 minutos para nitroanilinas), $1 \mathrm{mmol}$ de outra anilina foi adicionado diretamente à reação. Essa segunda anilina é adicionada sem solubilização prévia (isto é, conforme se apresenta, sólida ou líquida) e isso não apresenta problemas para a reação. Ela será eventualmente consumida na reação.

O banho de gelo foi então imediatamente removido e após a reação atingir a temperatura ambiente, a reação foi agitada por mais duas horas ou até a formação de um precipitado.

A mistura reacional é passada para um filtro com placa sinterizada ou funil de Buchner e filtrado sob vácuo. O sólido é seco sob pressão reduzida. Após análise por CCD (cromatografia em camada delgada) o rendimento foi determinado e foi submetido à caracterização por RMN e EMAR.

\section{PERIGOS E PRECAUÇÕES}

Os alunos devem examinar a FISPQ de todos os produtos utilizados e submeterem-se aos procedimentos padrão de segurança de laboratório, com destaque para o uso de luvas, já que triazenos podem desencadear dermatite alérgica de contato em pessoas alérgicas. Todos os triazenos sintetizados neste trabalho possuem características corantes, o que dificulta sua remoção de tecidos. Anilinas possuem odor forte. Anilinas, triazenos e nitritos podem ser cancerígenos.

Nenhum composto facilmente inflamável, aquecimento, alta pressão ou gases tóxicos estão envolvidos na reação, entretanto, todas as reações devem ser feitas em capela de laboratório com exaustão sempre que possível.

\section{RESULTADOS E DISCUSSÃO}

\section{1,3-diariltriazenos simétricos}

Nesta primeira abordagem, investigamos a reação de formação de 1,3-diariltriazenos substituídos simétricos. De acordo com a literatura, a adição de meio equivalente de nitrito de sódio $\left(\mathrm{NaNO}_{2}\right)$ na anilina em meio ácido leva à formação do produto de interesse.4 Optamos pelo uso do vinagre, uma solução diluída de ácido acético, por ser prontamente disponível, de baixo custo e uma opção mais verde. Assim, observamos que na maioria das anilinas testadas houve a formação de um precipitado que foi prontamente separado por filtração à vácuo. Os rendimentos estão sumarizados na Tabela 1.

A formação de alguns dos triazenos foi confirmada através da análise de EMAR, resultando na massa e o padrão isotópico esperado para cada produto, da análise $\mathrm{RMN}{ }^{1} \mathrm{H}$ e determinação do ponto de fusão que foram comparadas com a literaturas, quando disponíveis. Os pontos de fusão, no geral, divergiram, provavelmente por não terem sido cristalizados os produtos. Tais dados estão presentes no Material Suplementar.

De acordo com os valores de rendimento da Tabela 1, os maiores rendimentos foram obtidos quando o grupo substituinte na anilina possui um maior caráter retirador de elétrons. Entretanto, se o efeito retirador é forte, como visto nas nitroanilinas, se torna necessário um maior tempo de reação, visto que o conhecido mecanismo de diazotação e o de formação do triazeno tem como a própria anilina sendo o nucleófilo. Ao diminuir a nucleofilicidade, a velocidade da reação tende a diminuir, por se tornar menos reativo. Tal explicação vai de acordo com os resultados obtidos, uma vez que foi necessária uma hora para a diazotação e cerca de 24 horas de reação para se obter uma boa conversão.

Outro fator a se destacar é o efeito da posição do grupo substituinte. A posição -orto apresenta rendimentos inferiores as demais posições, como pode ser visto comparando $\mathbf{2 c}\left(p-\mathrm{NO}_{2}\right) \operatorname{com} \mathbf{2} \mathbf{j}$ $\left(o-\mathrm{NO}_{2}\right)$ e $\mathbf{2 e}(p-\mathrm{Br}), \mathbf{2} \mathbf{h}(m-\mathrm{Br})$ com $\mathbf{2 i}(o-\mathrm{Br})$. Tal fato provavelmente se deve à problemas estéricos causados pela aproximação da porção nucleofílica.

Um ponto de extrema importância é diferença entre anilinas com grupos eletrodoadores e eletroretiradores. Enquanto as com grupos retiradores não apresentaram problemas na síntese inicialmente proposta (Método A), ao se encaminhar para a síntese utilizando as com grupos eletrodoadores (1a, 1f, $\mathbf{1 g}$ ) observou que além da formação do triazeno, havia a formação de outros subprodutos desconhecidos (monitorado via $\mathrm{CCD}$ ), porém não havia precipitado algum ao final da reação, necessitando uma etapa de extração líquidolíquido. Assim, foi preciso uma alteração na metodologia.

Buscando inspiração na literatura, foi observado que diversas metodologias de síntese de triazenos utilizam acetato de sódio durante a síntese.12 Dessa forma, o Método B foi designado para essas anilinas com grupos eletrodoadores, apresentando melhores resultados para $\mathbf{2}$ a e $\mathbf{2 f}$, porém, sem sucesso para $\mathbf{2 g}$, tanto em questão de pureza, quanto de precipitação do triazeno. A adição de acetato de sódio serve para tornar o meio mais básico pois testes com cloreto de sódio à reação não levaram à precipitação dos diariltriazenos de interesse, excluindo a hipótese de efeito salting-out. Desta forma, o meio fracamente ácido do vinagre é suficiente para decompor e também não favorecer a formação eficiente de triazenos oriundos de anilinas com grupos doadores de elétrons.

Testes submetendo os compostos solvatados separadamente em acetona, acetato de etila e clorofórmio, indicaram que solventes mais ácidos, como o o clorofórmio, tendem a degradar os triazenos, enquanto outros solventes não afetam o composto. Foi visto que triazenos oriundos de anilinas com grupos retiradores de elétrons apresentam maior estabilidade em meio ácido, sendo estáveis ou pouco decompostos quando submetidos a até quatro dias ao solvente. Triazenos de anilinas com grupos fracamente retiradores de elétrons sofreram maior degradação, em questão de horas, enquanto que $\mathbf{2 f}$ (fracamente doador) degradou em questão de minutos. A presença 
Tabela 1. Síntese de 1,3-diariltriazenos simétricos em vinagre ${ }^{\mathrm{a}}$

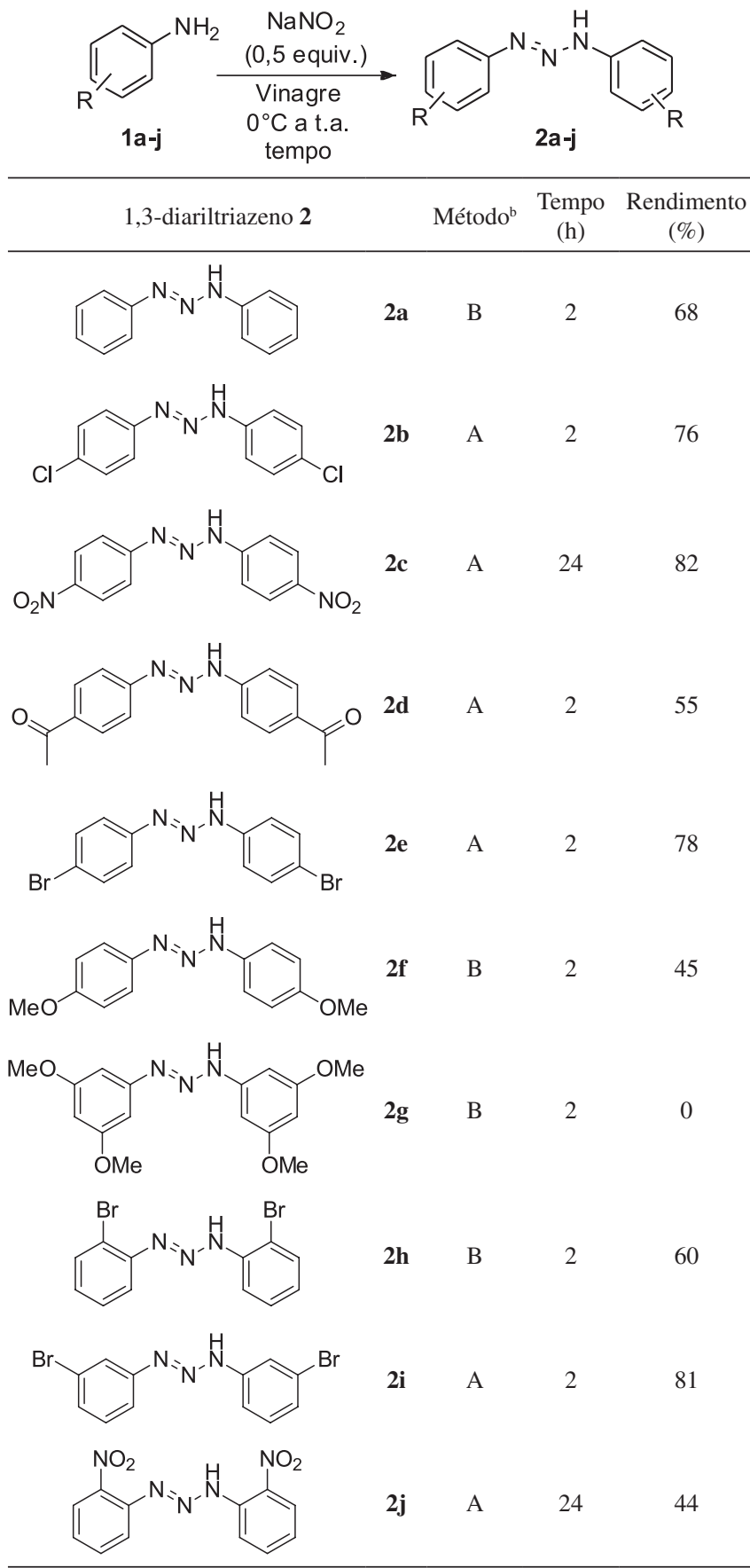

${ }^{\mathrm{a} C o n d i c ̧ o ̃ e s ~ e x p e r i m e n t a i s: ~ 1 a-j ~}(1 \mathrm{mmol})$, vinagre $30 \mathrm{~mL}$. ${ }^{\mathrm{b}}$ Método A: A reação foi mantida a temperatura ambiente por duas horas ou até a formação de um precipitado. Método B: Foi adicionado uma solução aquosa de acetato de sódio ( $15 \mathrm{~g} \mathrm{em} 20 \mathrm{~mL})$ e mantido sob agitação em temperatura ambiente por duas horas ou até a formação de um precipitado.

de degradação foi monitorada por CCD e é perceptível pelo escurecimento da solução. Tal resultado vai de acordo com estudos das literaturas que citam o mecanismo de decomposição e a tendência do $\mathrm{pKa}$ dos compostos. ${ }^{6,16}$

Uma adição ao experimento proposto é justamente esta verificação da decomposição dos triazenos. Caso tenha sido sintetizado o composto $\mathbf{2 f}$, adicionar uma ponta de espátula dele em eppendorfs ou tubos de ensaio separados contendo um pequeno volume de cloróformio e acetona possibilita visualizar a decomposição. O tubo

contendo clorofórmio irá escurecer em cerca de 10 a 30 minutos. Um placa de CCD pode ser corrida então, indicando a decomposição.

\section{1,3-diariltriazenos não-simétricos}

Para o estudo dos triazenos não-simétricos, a diazotação foi feita em anilinas que levavam a sais de diazônio estáveis que possam ser facilmente reagidos com a adição da segunda anilina. Assim, após a diazotação das orto e para nitroanilinas e da para cloroanilina, essas foram reagidas com outras anilinas. De maneira similar aos 1,3-diariltriazenos simétricos houve uma formação de um precipitado no meio reacional e uma simples filtração à vácuo levou aos 1,3-diariltriazenos não-simétricos. Os resultados estão sumarizados na Tabela 2.

Tabela 2. Resultados na formação de triazenos não-simétricos ${ }^{\mathrm{a}}$
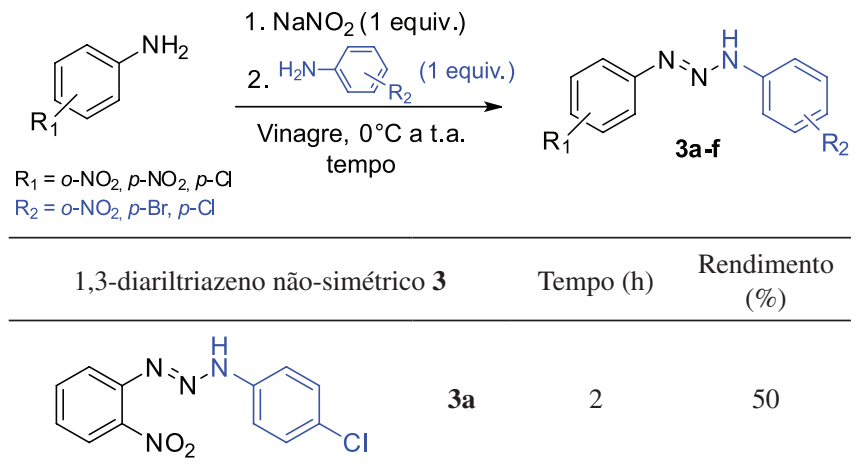

$3 a$

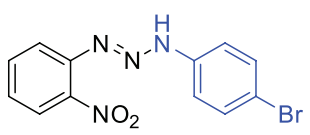

$3 \mathbf{b}$

2

6

64<smiles>O=[N+]([O-])c1ccc(/N=N/Nc2ccccc2[N+](=O)[O-])cc1</smiles><smiles>O=[N+]([O-])c1ccc(/N=N/Nc2ccc(Cl)cc2)cc1</smiles>
3d 2<smiles>O=[N+]([O-])c1ccc(/N=N/Nc2ccc(Br)cc2)cc1</smiles>
3e 2 57

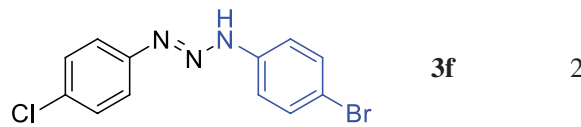

${ }^{\mathrm{a}}$ Condições experimentais: anilinas ( $1 \mathrm{mmol}$ cada), vinagre $30 \mathrm{~mL}$.

O triazeno $3 \mathbf{c}$ necessitou de seis horas de reação por se tratar de uma reação entre duas nitroanilinas, que assim como no caso de simétricos de nitroanilina, possuem uma menor reatividade no nucleófilo devido ao grupo fortemente eletroretirador, o que exige um maior tempo de reação frente as demais para que se obtenha um rendimento equiparável.

Verificamos também se a mudança da ordem das anilinas influenciaria no rendimento. No caso do triazeno 3a, por exemplo, ao se realizar a diazotação da para-cloroanilina seguida da adição da orto-nitroanilina, percebemos uma diminuição na velocidade da formação do produto devido à menor nucleofilicidade e, portanto, reatividade da nitroanilina. Após duas horas de reação o rendimento obtido foi de $4 \%$, contra os $50 \%$. Assim, tornou-se evidente que é 
preferível diazotar a anilina com grupo mais fortemente retirador primeiramente, para, em seguida, adicionar à reação a outra anilina, com grupo menos retirador, que atuará como um nucleófilo mais eficiente.

Quanto à elucidação por RMN, o hidrogênio ligado ao nitrogênio do grupo triazo aparece em sinal desblindado variando de 9 a 12 ppm geralmente. Um sinal a mais é visualizado na maioria dos espectros de $\mathrm{RMN}{ }^{1} \mathrm{H}$ na região de $\mathrm{HOD}$ (1,62 ppm em $\mathrm{CDCl}_{3} ; 2,83$ ppm em acetona- $\mathrm{d}_{6}$ ), que embora possa parecer problemas na secagem do triazeno sólido, na verdade a hipótese é de que seja água de hidratação presentes na estrutura do composto, uma vez que as integrais destes sinais de HOD apresentam-se como múltiplos $(0,5 ; 1,0 ; 1,5 ; 2,0)$. Entretanto, não foi realizada nenhuma caracterização que comprove isso. Porém, vale lembrar que os compostos foram submetidos a no mínimo oito horas sob vácuo de bomba de alto vácuo e foi certificado nas últimas horas que não havia mais variação de massa e, portanto, não deveria haver mais excesso de água a ser eliminado do composto. Não foram encontrados indícios na literatura que corroborem essa teoria. Espectros de RMN apresentado em outros trabalhos não sugerem a presença desse sinal, mas geralmente são sintetizados em meio orgânico ou incluída a etapa de recristalização do triazeno, o que poderia eliminar essa susposta água de hidratação nesses casos.

\section{CONCLUSÕES}

Ao que cabe ao trabalho, foi possível prover uma síntese bastante simples, rápida, genérica e verde de 1,3-diariltriazenos de boa reprodutibilidade, de médio a alto rendimento e de elevada pureza, principalmente para anilinas com grupos substituintes fracamente retiradores até fortemente retiradores. Para anilinas contendo grupos eletrodoadores foi possível identificar a decomposição em meio ácido de seus triazenos, além da maior tendência a não precipitar. Contudo, para contornar isso, a alteração do $\mathrm{pH}$ do meio foi indicado para prevenir a decomposição e promover a precipitação.

$O$ efeito da ordem da adição das anilinas na produção de nãosimétricos foi brevemente verificada, chegando-se à conclusão de que, para fins de pureza, rendimento e tempo, é indicado diazotar a anilina com o grupo mais fortemente retirador. Estudos da estabilidade dos compostos em prolongada exposição à solvente ácido foram feitos por CCD e relacionados com informações da literatura, indicando uma influência positiva na estabilidade em triazenos com grupos substituintes eletroretiradores nas arilas.

Acredita-se que devido à facilidade e rapidez do procedimento experimental e toda a discussão que é possível construir acima dos resultados destes compostos, a prática apresentada é uma boa opção para ser executada em um laboratório de graduação.

\section{MATERIAL SUPLEMENTAR}

O Material Suplementar se encontra em http://quimicanova.sbq. org.br e contém a descrição do sólido, ponto de fusão, espectro de RMN ${ }^{1} \mathrm{H}$ e espectro de EMAR, bem como as referências encontradas, quando disponíveis, para todos os compostos sintetizados. Também são fornecidas questões que podem ser propostas para os estudantes, além de anotações para professores.

\section{AGRADECIMENTOS}

À UFABC pela estrutura e à Central Experimental Multiusuário da UFABC pelos equipamentos.

\section{REFERÊNCIAS}

1. Perry, M. J.; Carvalho, E.; Rosa, E.; Iley, J.; Eur. J. Med. Chem. 2009, 44, 1049

2. Paraginski, G. L.; Berticelli, C. R.; Zambiazi, P. J.; Paraginski, V. T. K.; Hörner, M.; dos Santos, A. J. R. W. A.; Hörner, R.; Quim. Nova 2014, 37, 1138 .

3. Hörner, M.; Giglio, V. F.; dos Santos, A. J. R. W. A.; Westphalen, A. B.; Iglesias, B. A.; Martins, P. R.; Amaral, C. H.; Michelot, T. M.; Reetz, L. G. B.; Bertoncheli, C. M.; Paraginski, G. L.; Hörner, R.; Rev. Bras. Cienc. Farm. 2008, 44, 442.

4. Giglio, V. F. Tese de doutorado, Universidade Federal de Santa Maria, Brasil, 2011.

5. Kuriakose, S.; Muleme, H. M.; Onyilagha, C.; Singh, R.; Jia, P.; Uzonna, J. E.; PloS One 2012, 7, 1

6. Lim, H.; Zhang, H.; Barra, M.; J. Chem. Eng. Data 2010, 55, 4917.

7. Kimball, D. B.; Haley, M. M.; Angew. Chem., Int. Ed. 2002, 41, 3338.

8. Vernin, G.; Metzger, J.; Synthesis 1978, 12, 921.

9. Capeletto, M. G.; Filho, R. E. O.; Dionisio, V. Y.; Omori, A. T.; Curr. Green Chem. 2017, 4, 108.

10. Hino, K. N.; Omori, A. T.; Quim. Nova 2015, 38, 156.

11. Hill, D. T.; Stanley, K. G.; Williams, J. E. K.; Loev, B.; Fowler, P. J.; McCarfferty, J. P.;

Mackow. E.; Berkoff, C. E.; Ladd, C. B.; J. Med. Chem. 1983, $26,865$.

12. Vernin, G.; Siv, C.; Metzger, J.; Synthesis 1977, 10, 691.

13. Tundo, P.; Loris, A.; Selva, M.; Green Chem. 2007, 9, 777.

14. Bräse, S.; Gil, C.; Knepper, K.; Zimmerman, V.; Angew. Chem., Int. Ed. 2005, 44, 5188

15. Miller A.; Solomon, P. H.; Writing Reaction Mechanisms in Organic Chemistry, $2^{\text {nd }}$ ed., Academic Press: New York, 2000.

16. Chen, N.; Barra, M.; Lee, I; Chahal, N.; J. Org. Chem. 2002, 67, 2271. 